\title{
Combined Exp-Function Ansatz Method and Applications
}

\author{
Gui Mu, ${ }^{1}$ Jun Liu, ${ }^{1}$ Zhengde Dai, ${ }^{2}$ and Xi Liu ${ }^{3}$ \\ ${ }^{1}$ College of Mathematics and Information Science, Qujing Normal University, Qujing 655011, China \\ ${ }^{2}$ School of Mathematics and Statics, Yunnan University, Kunming 650091, China \\ ${ }^{3}$ School of Information Science and Engineering, Yunnan University, Kunming 650091, China
}

Correspondence should be addressed to Zhengde Dai; zhddai2004@yahoo.com.cn

Received 3 November 2012; Accepted 20 May 2013

Academic Editor: Syed Tauseef Mohyud-Din

Copyright (C) 2013 Gui Mu et al. This is an open access article distributed under the Creative Commons Attribution License, which permits unrestricted use, distribution, and reproduction in any medium, provided the original work is properly cited.

Our aim is to present a combined Exp-function ansatz method. This method replaces the traditional assumptions of multisolitons by a combination of the hyperbolic functions and triangle functions in Hirota bilinear forms of nonlinear evolution equation. Using this method, we can obtain many new type analytical solutions of various nonlinear evolution equations including multisoliton solutions as well as breath-like solitons solutions. These solutions will exhibit interesting dynamic diversity.

\section{Introduction}

Up to now, many kinds of integrable nonlinear partial differential equations have been discovered, such as nonlinear Schrodinger equation, KdV equation, Sine-Gordon equation, KP, BKP, coupled KP, and Toda lattice and Toda molecule equations. All of these equations can be transformed into bilinear forms by some special transformations including rational transformation, logarithmic transformation, and bilogarithmic transformation [1]. Once we get the bilinear forms of these equations, one can construct directly their $\mathrm{N}$-soliton solutions following Hirota's basic assumptions. In addition, bilinear forms can be utilized to construct the other kinds of solutions. Lou [2-6] has constructed many localized structures by a variable separation method, and the author of [1] has obtained determinants and pfaffians solutions using the bilinear forms. Recently, Dai et al. [7] proposed the three-wave method for nonlinear evolution equations (NEE). Meanwhile, some fractional differential equations and local fractional equations are studied extensively using different methods [8-10]. Analytical solutions for nonlinear partial differential equations are discussed systematically in [11]. Motivated by the above considerations, we investigate another ansatz and present "combined Exp-function ansatz method" as follows.
Consider a $(2+1)$-dimensional nonlinear evolution equation of the general form

$$
F\left(u, u_{t}, u_{x}, u_{y}, \ldots\right)=0,
$$

where $F$ is a polynomial of $u(x, y, t)$ and its derivatives. With the help of rational transformation, logarithmic transformation, and bilogarithmic transformation, for a KdV-type bilinear equation, it has just one dependent variable $f$. We next consider a bilinear equation of the form

$$
G\left(D_{t}, D_{x}, D_{y}, \ldots\right) f \cdot f=0,
$$

where $G$ is a general polynomial in $D_{t}, D_{x}, D_{y}$, where the $D$ operator is defined by

$$
\begin{aligned}
D_{x}^{m} D_{t}^{n} F(x, y, t) \cdot G(x, y, t) \\
=\left(\frac{\partial}{\partial x}-\frac{\partial}{\partial x^{\prime}}\right)^{m}\left(\frac{\partial}{\partial t}-\frac{\partial}{\partial t^{\prime}}\right)^{n} \\
\quad \times\left. F(x, y, t) G\left(x^{\prime}, y^{\prime}, t^{\prime}\right)\right|_{x^{\prime}=x, y^{\prime}=y, t^{\prime}=t^{\prime}}
\end{aligned}
$$

Traditionally, one obtains $N$-soliton solutions with the assumption

$$
f=\sum_{\mu=0,1} \exp \left(\sum_{i>j}^{N} A_{i j} \mu_{i} \mu_{j}+\sum_{i=1}^{n} \mu_{i} \xi_{i}\right) .
$$


Here, instead of the above assumption, the function $f$ is assumed in terms of cosh functions and cos functions

$$
\begin{aligned}
f= & \sum_{i=1}^{m} a_{i}\left(\exp \left(\xi_{i}\right)+\exp \left(-\xi_{i}\right)\right) \\
& +\sum_{j=1}^{n} b_{i}\left(\exp \left(i \xi_{j}\right)+\exp \left(-i \xi_{j}\right)\right),
\end{aligned}
$$

or equivalently

$$
f=2 \sum_{i=1}^{m} a_{i} \cosh \left(\xi_{i}\right)+2 \sum_{j=1}^{m} b_{i} \cos \left(\eta_{j}\right),
$$

where $\xi_{i}=k_{i} x+l_{i} y+c_{i} t$ and $\eta_{i}=d_{i} x+e_{i} y+f_{i} t$. In (5), it is seen that real and complex variables coexist in Exp-function; hence, this method is called combined Expfunction ansatz method. To derive analytic expression, we can take the following procedure in detail: inserting (5) into (2), then equating the coefficients of the same kind terms to zero, and subsequently solving the resulting algebraic equations to determine the relationship between variables $k_{i}, l_{i} \ldots$ with the help of symbolic computation software such as Maple. In (5), cosh functions are responsible for energy localization, but cosine functions take into account periodic effect in real physical background. If cosh functions and cosine functions coexist, the intensity of periodic effect depends on the scale distance between the coefficients $a_{i}$ and $b_{j}$. When all of the coefficients of cosine functions $b_{j}$ are equal to zero, (5) corresponds to multisoliton of (1).

\section{Application to $(2+1)$-Dimensional NLEE Equation}

In this section, firstly, we study the $(2+1)$-dimensional nonlinear evolution equation

$$
u_{x x x y}+3 u_{y} u_{x x}+3 u_{x} u_{x y}+2 u_{y t}=0 .
$$

In [12], Bekir has studied its Painlevé property. By the independent variable transformation $u=2(\ln \varphi)_{x x}$, (7) is reduced to Hirota bilinear form

$$
\left(D_{y} D_{t}+D_{x}^{3} D_{y}\right) \varphi \cdot \varphi=0 .
$$

Firstly, we obtain $N$-soliton with the aid of Hirota method. To get one-soliton solution, we assume that

$$
\phi=1+e^{k_{1} x+l_{1} y+c_{1} t}
$$

Inserting (9) into (8), then one-soliton solution can be derived as

$$
u(x, t)=\frac{2 k_{1} e^{k_{1} x+l_{1} y-k_{1}^{3} t}}{1+e^{k_{1} x+l_{1} y-k_{1}^{3} t}} .
$$

For the two-soliton solutions, substituting

$$
\phi=1+e^{k_{1} x+l_{1} y+c_{1} t}+e^{k_{2} x+l_{2} y+c_{2} t}+a_{12} e^{k_{1} x+l_{1} y+c_{1} t+k_{2} x+l_{2} y+c_{2} t}
$$

into (8) and solving for the phase shift $a_{12}$, one can find the two-soliton solutions explicitly. The higher level soliton solutions can be obtained in a parallel manner. Next, we will show how the combined Exp-function ansatz method is used to construct new exact solution of nonlinear evolution equation. In fact, the basic procedure is similar to $N$-soliton procedure. For simplification, we only present the case for the parameters $m=2$ and $n=1$ in (5) to explain our method. That is, we assume in the following form that

$$
\begin{aligned}
\varphi= & \cosh \left(k_{1} x+l_{1} y+c_{1} t\right)+\cos \left(k_{2} x+l_{2} y+c_{2} t\right) \\
& +a_{3} \cosh \left(k_{3} x+l_{3} y+c_{3} t\right) .
\end{aligned}
$$

Substituting (12) into (8), we have

$$
\begin{gathered}
c_{1}=-k_{3}^{3}\left(-1+3 l_{3}^{2}-6 l_{3}^{2} a_{3}^{2}+3 l_{3}^{4} a_{3}^{2}\right), \\
c_{2}=k_{3}^{3} l_{3}\left(1-a_{3}^{2}\right)\left(l_{3}^{2}-2 l_{3}^{2} a_{3}^{2}+a_{3}^{4} l_{3}^{2}-3\right), \\
c_{3}=k_{3}^{3}\left(-1+3 l_{3}^{2}-6 l_{3}^{2} a_{3}^{2}+3 a_{3}^{4} l_{3}^{2}\right), \\
k_{1}=-k_{3}, \quad k_{2}=l_{3} k_{3}\left(1-a_{3}^{2}\right), \\
l_{2}=1, \quad l_{1}=l_{3},
\end{gathered}
$$

where $l_{3}, a_{3}$, and $k_{3}$ are free parameters. This case leads to a breath-kink solitary solution

$$
\begin{gathered}
u(x, t)=\left(2 \left(k_{1} \sin \left(k_{1} x+l_{1} y+c_{1} t\right)\right.\right. \\
-k_{2} \sin \left(k_{2} x+l_{2} y+c_{2} t\right) \\
\left.\left.\quad+a_{3} k_{3} \sin \left(k_{3} x+l_{3} y+c_{3} t\right)\right)\right) \\
\times\left(\cosh \left(k_{1} x+l_{1} y+c_{1} t\right)+\cos \left(k_{2} x+l_{2} y+c_{2} t\right)\right. \\
\left.\quad+a_{3} \cosh \left(k_{3} x+l_{3} y+c_{3} t\right)\right)^{-1} .
\end{gathered}
$$

The dynamics of this family of solutions will breathe periodically in the process of propagation of the soliton resulting from cosine function. In order to explain the university of our method, next, we continue to consider the $(2+1)$ dimensional AKNS equation

$$
4 u_{x t}+u_{x x x y}+8 u_{x} u_{x y}+4 u_{y} u_{x x}=0 .
$$

Taking the transformation $u=(\ln \varphi)_{x}$, (15) leads to multibilinear form

$$
\begin{gathered}
\left(4 D_{x} D_{t}+D_{x}^{3} D_{y}\right) \varphi \cdot \varphi=0 \\
D_{x}(\ln f)_{x x} \cdot(\ln f)_{x y}=0 .
\end{gathered}
$$

According to the one-soliton assumption, the one-soliton solution of $(2+1)$-dimensional AKNS equation is derived as

$$
u(x, t)=\frac{k_{1} e^{k_{1} x+l_{1} y-(1 / 4) l_{1} k_{1}{ }^{2} t}}{1+e^{k_{1} x+l_{1} y-(1 / 4) l_{1} k_{1}{ }^{2} t}} .
$$


For the two-soliton solutions which can be obtained following the assumption in (16)

$$
\varphi=1+e^{k_{1} x+l_{1} y+c_{1} t}+e^{k_{2} x+l_{2} y+c_{2} t}+a_{12} e^{k_{1} x+l_{1} y+c_{1} t+k_{2} x+l_{2} y+c_{2} t},
$$

we have

$$
a_{12}=\frac{\left(k_{1}-k_{2}\right)\left(l_{2} k_{1}^{2}+2 l_{1} k_{1} k_{2}-2 l_{2} k_{1} k_{2}-l_{1} k_{2}^{2}\right)}{\left(k_{1}+k_{2}\right)\left(l_{2} k_{1}^{2}+2 l_{1} k_{1} k_{2}+2 l_{2} k_{1} k_{2}+l_{1} k_{2}^{2}\right)} .
$$

In addition,

$$
l_{1} k_{2}-l_{2} k_{1}=0
$$

Thus, we found the two-soliton solutions explicitly

$$
\begin{aligned}
u(x, t)= & \left(k_{1} e^{k_{1} x+l_{1} y-(1 / 4) l_{1} k_{1}{ }^{2} t}+k_{2} e^{k_{2} x+l_{2} y-(1 / 4) l_{2} k_{2}{ }^{2} t}\right. \\
& \left.+a_{12}\left(k_{1}+k_{2}\right) e^{k_{1} x+l_{1} y-(1 / 4) l_{1} k_{1}{ }^{2} t+k_{2} x+l_{2} y-(1 / 4) l_{2} k_{2}{ }^{2} t}\right) \\
\times & \left(1+e^{k_{1} x+l_{1} y-(1 / 4) l_{1} k_{1}{ }^{2} t}+e^{k_{2} x+l_{2} y-(1 / 4) l_{2} k_{2}{ }^{2} t}\right. \\
& \left.+a_{12} e^{k_{1} x+l_{1} y-(1 / 4) l_{1} k_{1}{ }^{2} t+k_{2} x+l_{2} y-(1 / 4) l_{2} k_{2}{ }^{2} t}\right)^{-1} .
\end{aligned}
$$

Similarly, the higher order soliton solutions can be examined in a parallel manner. Finally, following the procedure of combined Exp-function ansatz method, the two periodic solutions of AKNS equation can be obtained by setting $m=2$ and $n=1$ in (5) in the following form:

$$
\varphi=\cos \left(k x+l y+\left(2 k^{2} l-c\right) t\right)+\cos (k x+l y+c t),
$$

where $l$ and $k$ are free parameters. This case leads to a family of double periodic solutions as

$$
\begin{gathered}
u(x, t)=\left(-k \sin \left(k x+l y+\left(2 k^{2} l-c\right) t\right)\right. \\
+k \sin (k x+l y+c t)) \\
\times\left(\cos \left(k x+l y+\left(2 k^{2} l-c\right) t\right)\right. \\
+\cos (k x+l y+c t))^{-1} .
\end{gathered}
$$

The above solutions are given out for the first time in the literature.

\section{Conclusions}

Generally, $N$-soliton solution can be constructed after one obtains multilinear form of nonlinear evolution equations according to Hirota method. In this paper, we proposed a different ansatz method which is composed of complex and real exponential functions. This method allows us to construct multiple kinds of solutions, such as $N$-soliton solutions and breath-type solitary solutions. By taking two $(2+1)$-dimensional nonlinear evolution equations as examples, it is shown that this method is effective and direct for constructing new exact solutions of nonlinear integrable partial differential equations.

\section{Acknowledgments}

The work was supported by the Chinese Natural Science Foundation Grant no. 11061028, Yunnan NSF Grant no. 2010CD086, and Qujin Normal University NSF Grant no. 2010 QN018.

\section{References}

[1] R. Hirota, The Direct Method in Soliton Theory, vol. 155 of Cambridge Tracts in Mathematics, Cambridge University Press, Cambridge, UK, 2004.

[2] S.-Y. Lou, "Searching for higher-dimensional integrable models from lower ones via Painlevé analysis," Physical Review Letters, vol. 80, no. 23, pp. 5027-5031, 1998.

[3] S.-Y. Lou, "Dromions, dromion lattice, breathers and instantons of the Davey-Stewartson equation," Physica Scripta, vol. 65, no. 1, pp. 7-12, 2002.

[4] S.-Y. Lou, "On the coherent structures of the Nizhnik-NovikovVeselov equation," Physics Letters A, vol. 277, no. 2, pp. 94-100, 2000.

[5] S.-Y. Lou and H.-Y. Ruan, "Revisitation of the localized excitations of the $(2+1)$-dimensional KdV equation," Journal of Physics. A. Mathematical and General, vol. 34, no. 2, pp. 305316, 2001.

[6] S.-Y. Lou, J. Lin, and X.-Y. Tang, "Painlevé integrability and multi-dromion solutions of the $2+1$ dimensional AKNS system," The European Physical Journal. B. Condensed Matter Physics, vol. 22, no. 4, pp. 473-478, 2001.

[7] Z. D. Dai, C. J. Wang, S. Q. Lin, D. L. Li, and G. Mu, "The threewave method for nonlinear evolution equations," Nonlinear Science Letters A, vol. 1, no. 1, pp. 77-82, 2010.

[8] H. Jafari and H. Tajadodi, "He's variational iteration method for solving fractional Riccati differential equation," International Journal of Differential Equations, vol. 2012, Article ID 764738, 8 pages, 2010 .

[9] K. Li, J. Peng, and J. Jia, "Cauchy problems for fractional differential equations with Riemann-Liouville fractional derivatives," Journal of Functional Analysis, vol. 263, no. 2, pp. 476-510, 2012.

[10] J. J. Mohan and G. V. S. R. Deekshitulu, "Fractional order difference equations," International Journal of Differential Equations, vol. 2012, Article ID 780619, 11 pages, 2012.

[11] R. Ellahi and A. Zeeshan, Analytical Solutions for Nonlinear Partial Differential Equations, LAMBERT Academic Publishing GmbH, Saarbrücken, Germany, 2012, http://www.amazon .com/Analytical-Solutions-non-Linear-Differential-Equations/ $\mathrm{dp} / 3846524158$.

[12] A. Bekir, "Painlevé test for some $(2+1)$-dimensional nonlinear equations," Chaos, Solitons and Fractals, vol. 32, no. 2, pp. 449455, 2007. 


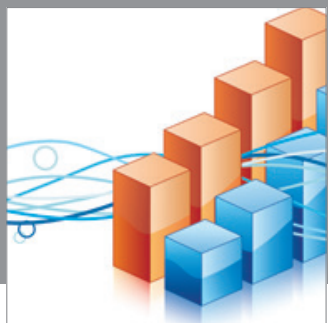

Advances in

Operations Research

mansans

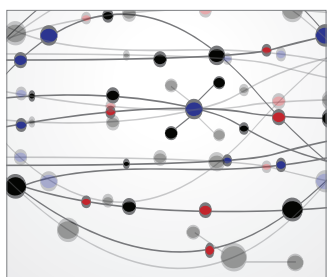

The Scientific World Journal
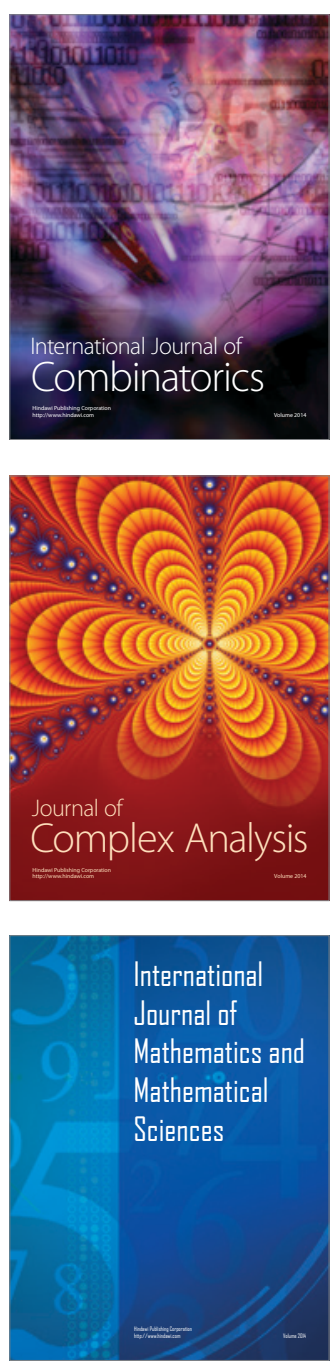
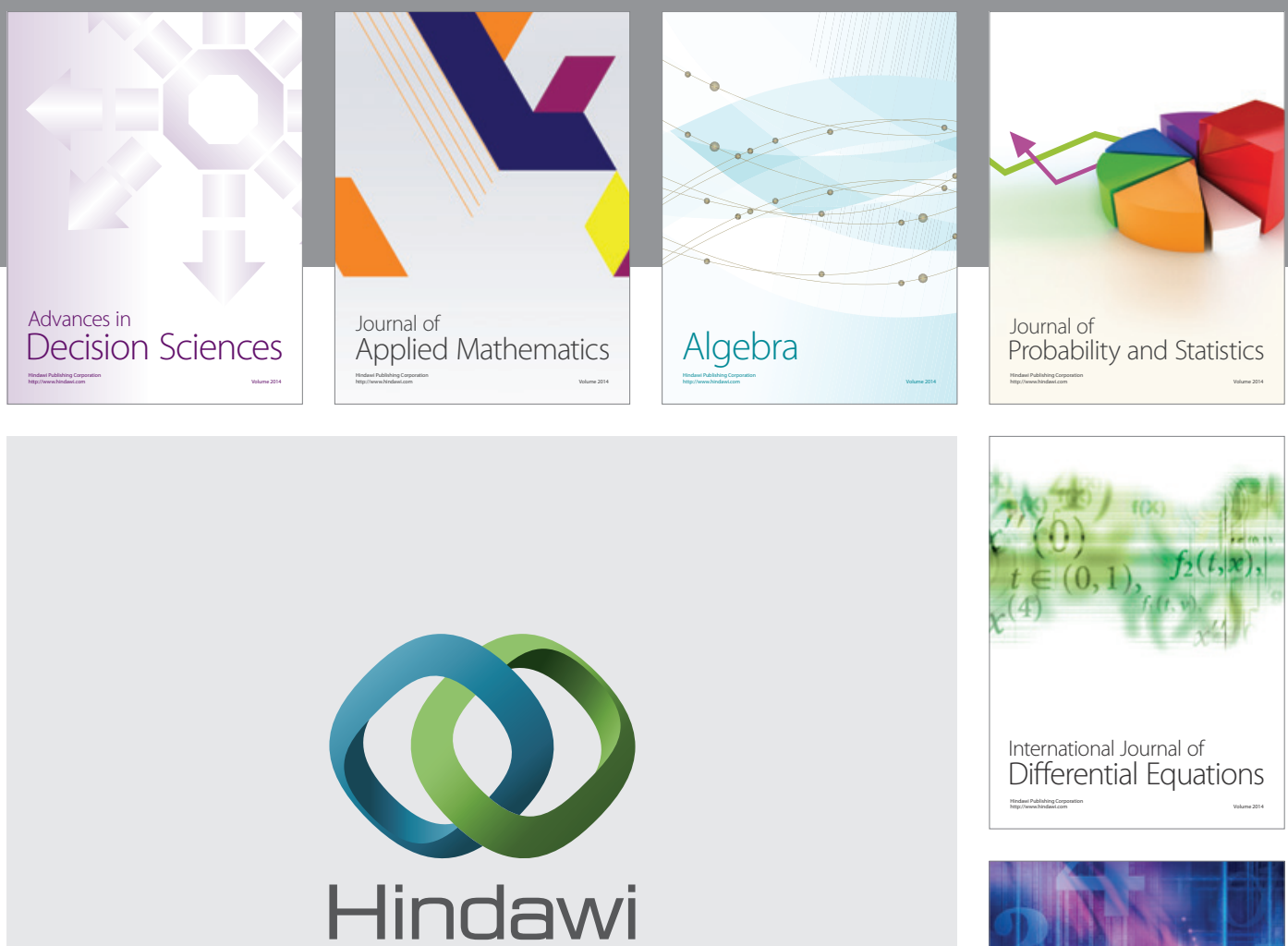

Submit your manuscripts at http://www.hindawi.com
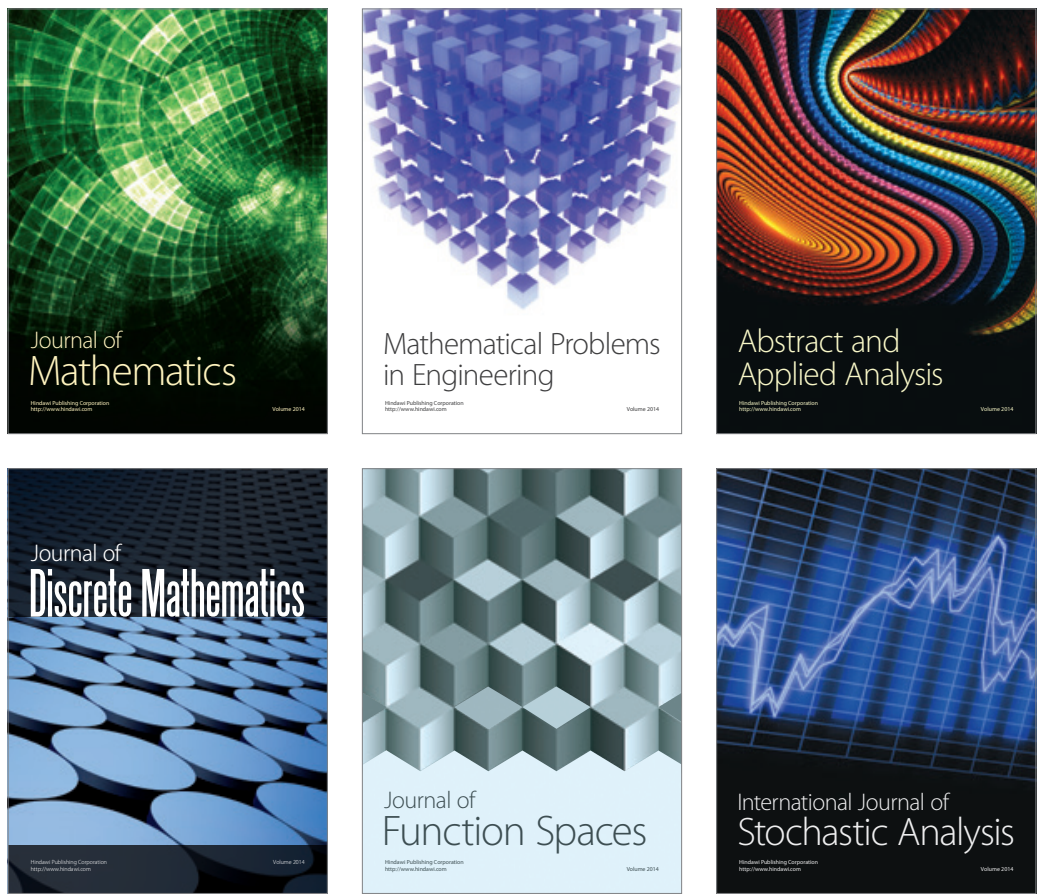

Journal of

Function Spaces

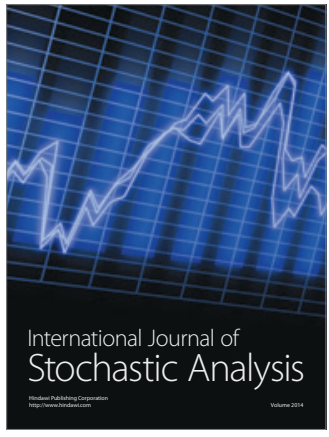

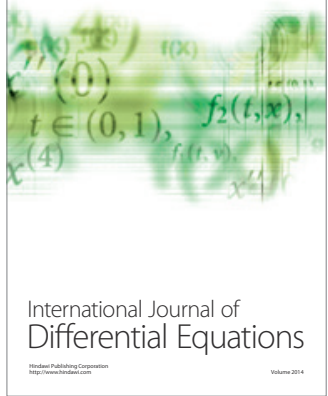
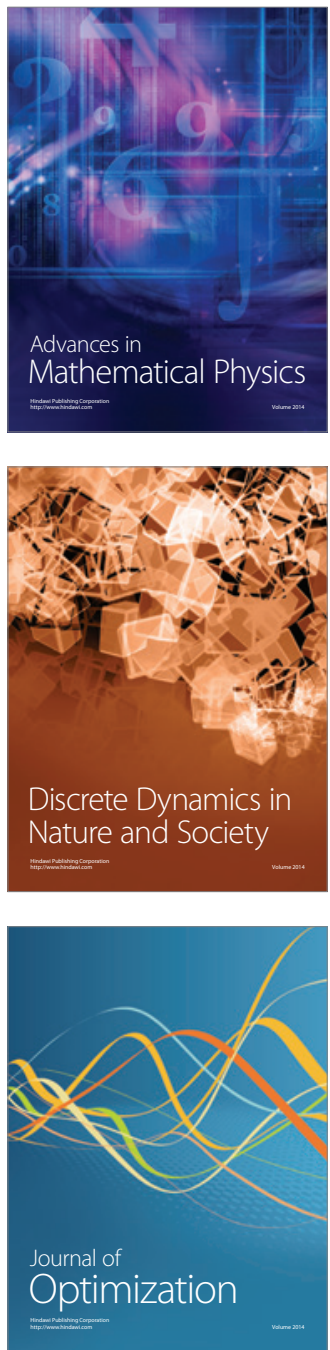